\title{
A Importância do Ginecologista na Prevenção do Câncer de Mama
}

\author{
Lívia Zancanella Dias \\ Universidade Severino Sombra, Acadêmica de Medicina, \\ lizancanella@gmail.com \\ Lucas Vinícius Silva Neves \\ Universidade Severino Sombra, Acadêmico de Medicina \\ lucas-vini@hotmail.com \\ Daniel de Araújo Dias \\ Universidade Severino Sombra, Academico de Medicina \\ dekar.lol@gmail.com \\ Henrique Erthal \\ Universidade Severino Sombra, Academico de Medicina \\ erthal.h@hotmail.com \\ Carlos Eduardo Cimatti Paulino \\ Universidade Severino Sombra, Academico de Medicina, \\ carloscp01@hotmail.com \\ Carla Vanúzia Braga Miranda Moura \\ Universidade Severino Sombra, Médica \\ em Radiologia e Diagnóstico por Imagem \\ carlavanuziamoura@gmail.com
}

\begin{abstract}
Resumo: O câncer de mama é a principal neoplasia maligna que acomete o sexo feminino no Brasil. O Profissional ginecologista é aquele que mais comumente está em contato com pacientes que possam vir a apresentar fatores de risco para o câncer de mama, e sua participação é imprescindível tanto na orientação quanto na prevenção dessa patologia. O presente trabalho tem por objetivo estudar a frequência do conhecimento e da realização do AEM, bem como ressaltar a importância da consulta ginecológica para e redução da incidência do câncer de mama.
\end{abstract}

Palavras-chave: Autoexame das mamas. Câncer de mama. Ginecologista câncer. Rastreamento.

Revista de Saúde, Vassouras, v. 3, n. 1, p. 5-12, jan./jun., 2012 


\title{
The Importance of the Gynecologist on the Breast Cancer Prevention
}

\begin{abstract}
Breast cancer is the leading cancer in Brazil. The professional gynecologist is one closest to patients who may have risk of Breast Cancer factors, and their participation both is essential guidance on the prevention of this disease. The present work aims to study the frequency of knowledge and the realization of the EMEA, as well as underscoring the importance of gynecological and the reduction of breast cancer incidence.
\end{abstract}

Keywords: Self-breast examination. Breast cancer. Gynecology. Cancer. Screening.

\section{Introdução}

O câncer consiste em uma enfermidade crônica, caracterizada pelo crescimento celular desordenado, o qual é resultante de alterações no código genético. Entre 5\% a 10\% das neoplasias são resultados diretos da herança de genes relacionados ao câncer, mas grande parte envolve danos ao material genético, de origem física, química ou biológica que se acumulam ao longo da vida (WCRF, 2007; Adami et al, 2001).

Atualmente, o câncer de mama apresenta elevada incidência e mortalidade em todo o mundo, representando um importante problema de saúde pública. O câncer de mama permanece como o segundo tipo de câncer mais frequente no mundo e o primeiro entre as mulheres. No Brasil, estima-se um número de 48.930 casos novos de câncer de mama em 2006, com um risco estimado de 52 casos a cada 100 mil mulheres (Felden et al, 2011). Essa patologia vem atingindo progressivamente um número maior de mulheres, em faixas etárias mais baixas, e com taxa de mortalidade também crescente no país (Sclowitzet al, 2005).

As formas mais eficazes para detecção precoce do câncer de mama são o autoexame das mamas (AEM), o exame clínico e a mamografia. A mamografia e ultrassonografia identificam tumores não palpáveis, apresentam alto custo e não fornecem resultados operacionais para serem aplicados em grandes massas populacionais. Consagra-se o AEM mensal como estratégia de escolha, uma vez que se caracteriza como prevenção secundária, sem custos e segura. As pesquisas indicam o impacto significativo do AEM na detecção precoce do câncer de mama, pois este registra tumores primários menores e menor número de linfonodos axilares invadidos pelo tumor (ou por células neoplásicas) nas mulheres que fazem o exame regularmente, além de haver também detecção de pequenas mudanças nas propriedades físicas das mamas, diminuindo assim, a probabilidade de metástase e aumentando a sobrevida. Estudos demonstram que a sobrevida em cinco anos tem sido de $75 \%$ entre as praticantes do AEM contra $57 \%$ entre as não praticantes (Felden et al, 2011).

A consulta médica de maneira geral tem grande importância na vida dos pacientes. No caso das mulheres, a consulta ginecológica é fundamental à prevenção do câncer, 
especialmente, o cérvico-uterino e o de mama, sendo também indispensável em programas de planejamento familiar, pré-natal, atendimento a patologias obstétricas e controle de doenças sexualmente transmissíveis [Torres et al, 2008].

Assim, objetiva-se estudar a frequência do conhecimento e da realização do AEM, bem como ressaltar a importância da consulta ginecológica para e redução da incidência do câncer de mama.

\section{Materiais e Métodos}

Estudo transversal de base populacional realizado por meio de questionário preenchido em entrevista por mulheres na faixa etária superior a 15 anos. Para a definição da casuística, utilizou-se como critério a faixa etária citada na literatura. A respeito da pergunta "Sabe como fazer o autoexame das mamas?", este conhecimento referia-se ao fato de a entrevistada saber ou não como se realiza o mesmo.

Em termos da realização do exame pelo médico ginecologista e o seu incentivo à prática do autoexame, foram consideradas todas as mulheres entrevistadas que referiram ter ido a pelo menos uma consulta ginecológica. Foram coletadas informações sobre variáveis demográficas, comportamentais, biológicas e referentes ao manejo médico. As entrevistadas faziam parte da demanda espontânea do Hospital Universitário Sul Fluminense e, igualmente, em feiras de saúde realizadas, no presente ano, na cidade de Vassouras - RJ, o que perfez um total de 266 entrevistas. Todas as pacientes assinaram termo de consentimento livre e esclarecido antes da realização das entrevistas.

As informações foram inseridas em banco de dados do software SPSS versão 19. As tabelas e gráficos foram construídos, respectivamente, pelos softwares Microsoft Excel 2007 e Microsoft PowerPoint 2007.

\section{Resultados}

Dos 266 questionários, foi observado que quase a totalidade das entrevistadas $(95,9 \%)$ já se consultou com um ginecologista (Gráfico 1), tendo em vista que a faixa etária entre 20 e 40 anos constitui a maior parte sobre a que faz o AEM regularmente $(46,9 \%)$.

Também se observou que das 266 mulheres entrevistadas, apenas 33,08\% sabiam como fazer o AEM (Gráfico 2). Em relação à faixa etária, mulheres entre 20 e 40 anos são as que mais obtêm o conhecimento sobre como se fazer o AEM (52,2\%).

Grande parte das entrevistadas $(92 \%)$ realizou ao menos uma consulta ginecológica no ano corrente ou no último ano (Gráfico 3).

Foi visto que $71,8 \%$ das entrevistadas receberam orientação por parte dos médicos sobre autoexame das mamas na última consulta ginecológica (Gráfico 4). 
Gráfico 1. Índice de mulheres que se consultaram com ginecologista.

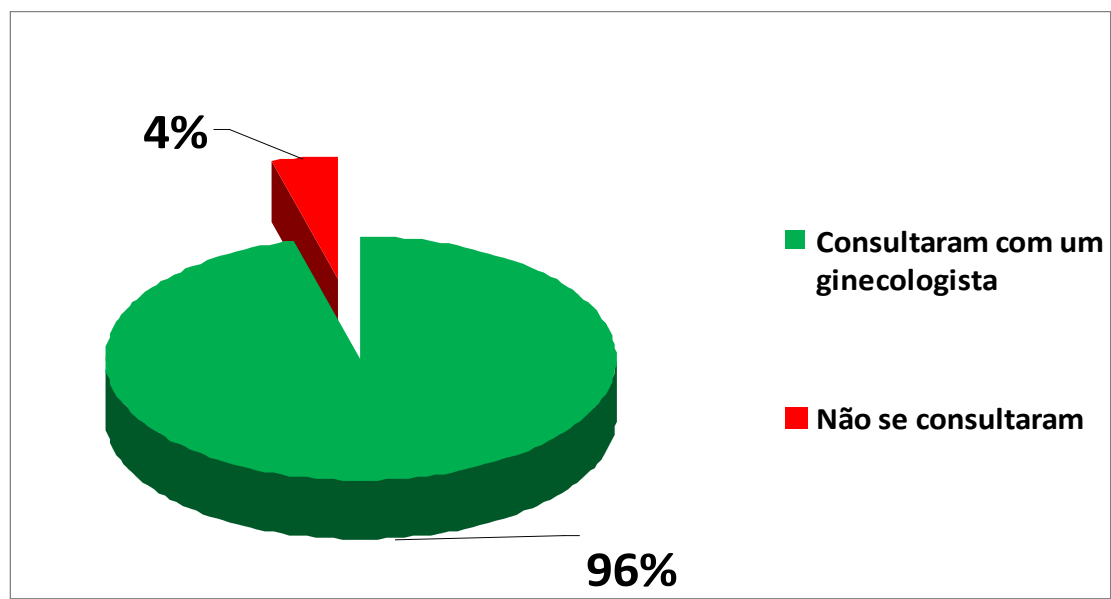

Gráfico2. Índice de mulheres que sabiam fazer o AEM.

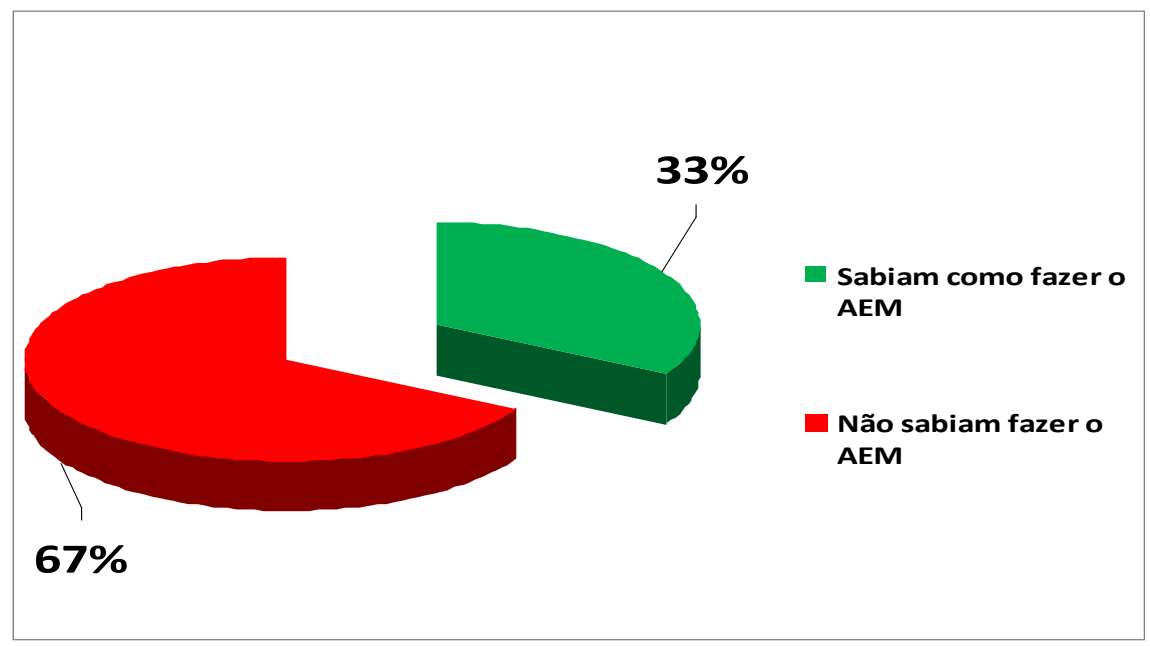

Revista de Saúde, Vassouras, v. 3, n. 1, p. 5-12, jan./jun., 2012 
Gráfico 3. Índice de mulheres que se consultaram com ginecologista no ano corrente ou no último ano.

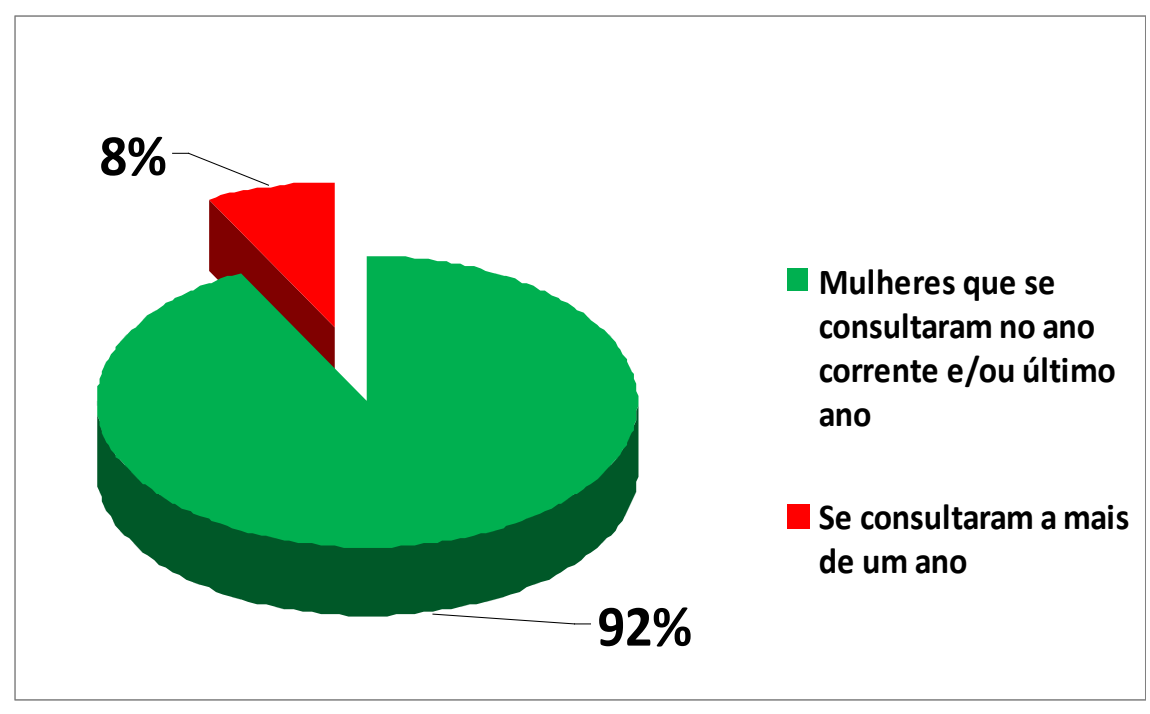

Gráfico 4. Índice de mulheres que receberam orientação dos médicos sobre o autoexame das mamas

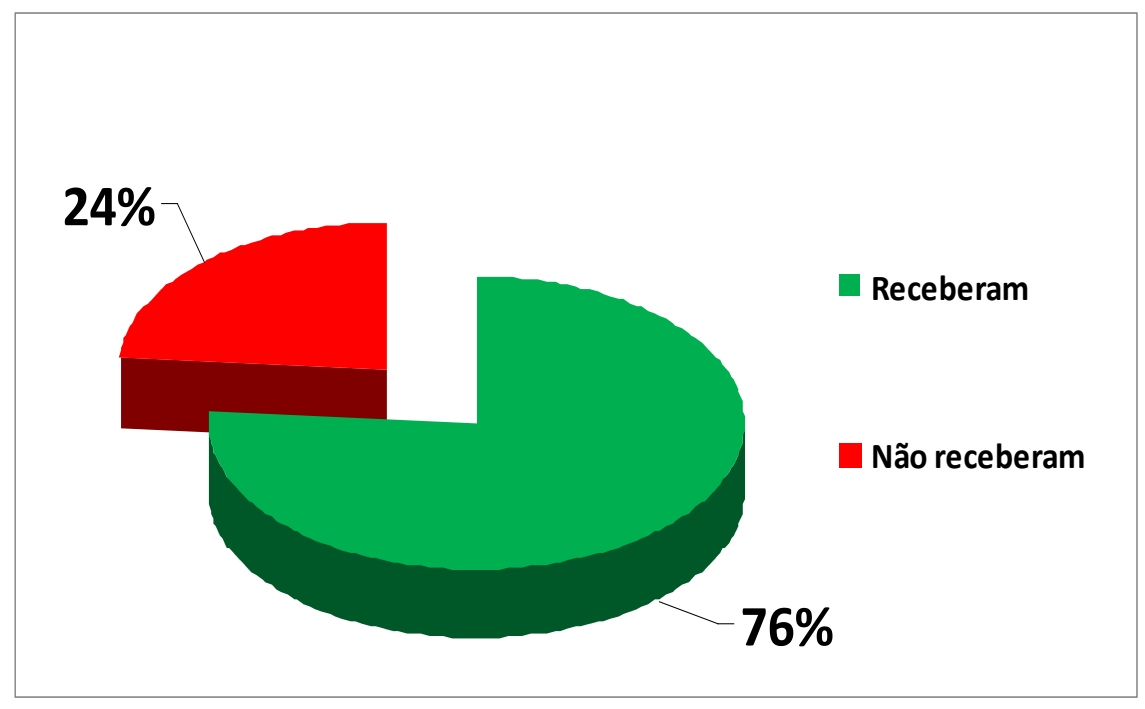

Revista de Saúde, Vassouras, v. 3, n. 1, p. 5-12, jan./jun., 2012 


\section{Discussão}

Vários métodos empregados para o diagnóstico precoce do câncer de mama, como a mamografia e ultrassonografia, que identificam tumores não palpáveis, menores que $1 \mathrm{~cm}$, apresentam alto custo e não fornecem resultados operacionais para serem aplicados em populações. Dessa forma, consagra-se o AEM mensal, inserido num processo educativo, juntamente com o exame das mamas por profissional treinado, como estratégia de escolha. Por isso, tornou-se importante estudar a frequência do conhecimento e prática do AEM (MTC et al, 1990).

As evidências de que o câncer pode ser prevenido vêm de estudos internacionais em que se observaram variações nas taxas de incidência de câncer segundo as variáveis, tempo e lugar. Populações que migram do país de origem para outras localidades apresentam mudanças nas taxas de incidência de câncer, o que demonstra que as neoplasias são, em parte, influenciadas pelas condições ambientais, não sendo determinadas apenas por fatores genéticos (AC, 2005).

Segundo Instituto Nacional de Câncer (INCA), pelo menos um terço de casos novos de câncer que ocorrem no mundo anualmente, poderiam ser prevenidos (Instituto Nacional de Câncer, 2009).

De acordo com estudos feitos por Freitas Júnior et al, apenas um terço das mulheres que conheciam o AEM realizavam-no de forma sistemática. A frequência de realização do autoexame influencia diretamente sua acurácia (Borba AA et al, 1998). Para mulheres que nunca praticaram o AEM, geralmente os nódulos cancerígenos identificados medem $3,5 \mathrm{~cm}$, para as que o praticam eventualmente, os nódulos têm aproximadamente $2,5 \mathrm{~cm}$ e, para as que o fazem mensalmente, são identificados com aproximadamente $2 \mathrm{~cm}$ ou menos. Aquelas que praticam o autoexame e descobrem nódulos, têm expectativa de vida de $75 \%$ e as que não o fazem reduzem suas chances para 59\% (MTC et al, 1990).

Entretanto, também devemos nos preocupar com o percentual de mulheres que não estão incluídas neste grupo. O papel do profissional de saúde é fundamental para orientar as mulheres quanto à frequência das consultas ginecológicas e quanto à importância em realizar periodicamente exames de deteç̧ão precoce como a mamografia, o exame clínico das mamas e o autoexame (Santos et al, 2001). É importante ressaltar que a detecção da doença em estágio inicial favorece tratamentos que podem erradicar totalmente o câncer de mama (Lima et al, 2011).

Não podemos deixar de citar que, de acordo com estudos realizados, e tendo em conta algumas das várias lições de experiências bem-sucedidas de países que conseguiram sucessos na redução da mortalidade por câncer de mama (Reino Unido, Canadá, Japão, Austrália e EUA, entre outros) pode-se notar que o principal desafio para os países latino-americanos consiste em desenvolver programas abrangentes para o tratamento do câncer de mama, devidamente estruturado e com recursos adequados (Robledo et al, 2010).

De acordo com os resultados encontrados no presente estudo, a maioria das mulheres está apta a realizar o auto-exame das mamas de forma consciente reafirmando assim a importância do ginecologista em educação em saúde, principalmente na prevenção do câncer de mama. 


\section{Conclusão}

O diagnóstico precoce do câncer de mama está ligado, indubitavelmente, ao acesso à informação para as mulheres, conscientizando-as sobre a realização do autoexame da glândula mamária, da busca pelo exame clínico e pela mamografia, tríade na qual deve se basear o rastreamento dessa neoplasia (Júnior R et al, 1996).

É importante que a detecção precoce do câncer de mama por meio do ensino do autoexame seja de responsabilidade de todos os que assistem a pacientes do sexo feminino, e não apenas daqueles que atuam em programas específicos para esse fim $^{12}$. É conhecido que o momento do diagnóstico do câncer tem influência direta em sua evolução e prognóstico, sendo a precocidade do diagnóstico diretamente proporcional às chances de cura (JRM, 2006; Piato S, 2002).

A prevenção e a promoção devem ser prioritárias em relação à saúde da mulher, principalmente quando se refere ao câncer que mais acomete a população feminina, o de mama. O ginecologista tem fundamental importância na orientação de suas pacientes quanto a essa terrível patologia. Desse modo, é interessante a propagação da informação de forma mais abrangente possível, antes que seja tarde, antes que seja ontem. 


\section{Bibliografia}

Adami HO et al (2001). Primary and secondary prevention in the reduction of cancer morbidity and mortality. Eur J Cancer; 37 Suppl 8: S118-27.

Ana Lívia Pontes de Lima et al (2011). Opportunistic screening for breast cancer among young women in Maranhão State, Brazil. Cad. Saúde Pública, Rio de Janeiro, 27(7): 1433-1439, jul.

Ana Paula de Sousa Monteiro et al. Breast Self-examination: Frequency of Knowledge, Practice and Associated Factors. RBGO 25 (3): 201-205, 200.

Borba AA et al (1998). Frequência de realização e acurácia do autoexame das mamas na detecção de nódulos em mulheres submetidas à mamografia. Rev. Bras. Ginecol Obstet; 20:37-43.

Freitas Júnior R et al (1996). Fatores determinantes do conhecimento e prática do autoexame de mama. Rev Bras Ginecol Obstet; 18:387-91.

Glenda Dias dos Santos et al (2001). Awareness about breast câncer and mammography in elderly women who frequent Daycare Centers in São Paulo (SP, Brazil). Ciência \& Saúde Coletiva, 16(5): 2533-2540.

Graham AC (2005). Epidemiology and prevention of breast cancer. Cancer Epidemiol Biomarkers Prev; 14:768-72.

Instituto Nacional de Câncer (2009). Estimativa 2010: incidência de câncer no Brasil. Rio de Janeiro: Instituto Nacional de Câncer.

Jussara Beatriz Borre Felden et al (2011). Distribution of body fat and breast cancer: a case-control study in the South of Brazil. Felden JBB, Figueiredo ACL.

Laganá MTC et al (1990). Auto exame de mama: identificação dos conhecimentos, atitudes, habilidades e práticas (CAHP) requeridos para elaboração de propostas educativas. Rev. Esc. Enfermagem USP; 24:281-99.

Luz María González-Robledo et al (2010). Acciones gubernamentales para la detección temprana del cáncer de mama en América Latina. Retos a futuro. Salud pública de méxico / vol. 52, no. 6, noviembre-diciembre..

Marcelo Leal Sclowitz et al (2005). Condutas na prevenção secundária do câncer de mama e fatores associados. Rev Saúde Pública, 39(3):340-9.

Maria Eponina de Abreu e Torres et al (2008). "Vai lá, tira a roupa... e... pronto...": o acesso a consultas ginecológicas em Belo Horizonte, MG. R. Bras. Est. Pop. São Paulo, v. 25, n. ${ }^{\text {o }} 1$, p. 49-69, jan./jun.

Piato S, Piato JRM (2006). Doenças da mama. 1. a ed. Rio de Janeiro: Revinter.

Piato S (2002). Tratado de Ginecologia. 2. ${ }^{\text {a }}$ ed. São Paulo: Artes Médicas.

World Cancer Research Fund/American Institute for Cancer Research (2007). Food, nutrition, physical activity, and the prevention of cancer: a global perspective. Washington DC: American Institute for Cancer Research. 